\title{
Commentary: Cell proliferation and immune evasion: A dangerous combination
}

\author{
Matthew J. Bott, MD \\ From the Thoracic Service, Department of Surgery, Memorial Sloan Kettering Cancer Center, New York, NY. \\ Disclosures: Author has nothing to disclose with regard to commercial support. \\ Received for publication May 21, 2019; accepted for publication May 23, 2019; available ahead of print July 10, \\ 2019. \\ Address for reprints: Matthew J. Bott, MD, Thoracic Service, Department of Surgery, Memorial Sloan Kettering \\ Cancer Center, 1275 York Ave, C-875, New York, NY 10065 (E-mail: bottm@mskcc.org). \\ J Thorac Cardiovasc Surg 2019;158:920-1 \\ $0022-5223 / \$ 36.00$ \\ Copyright (c) 2019 Published by Elsevier Inc. on behalf of The American Association for Thoracic Surgery \\ https://doi.org/10.1016/j.jtcvs.2019.05.079
}

T-cell checkpoint inhibition has become an important treatment option for patients with lung cancer. Although a subset of patients have demonstrated durable disease control following treatment, predicting these responses using clinical and pathologic data remains challenging. Mitchell and colleagues ${ }^{1}$ report an interesting association between cellular proliferation, as measured by Ki67 expression, and immune checkpoint expression in patients with stage I non-small cell lung cancer (NSCLC).

Using immunohistochemical analysis of tissue microarrays containing samples from 190 resected NSCLC specimens, the authors demonstrate that Ki67 expression was positively correlated with expression of immunosuppressive proteins such as programmed death ligand-1 (PD-L1), B7-H3, and lymphocyte activating 3 (LAG3). Similarly, they show that tumors with relatively high Ki67 expression had lower intratumoral density of certain immune cell subsets, which suggests that increased cellular proliferation is associated with an immunosuppressive microenvironment in early-stage cancers. Furthermore, this association could have significant implications for patient outcomes, because Ki67 expression was independently associated with worse overall survival in patients with stage I NSCLC. Although the current study does not directly demonstrate that Ki67 expression predicts response to T-cell checkpoint inhibition, this will presumably be explored in further studies by the authors.

The association described by Mitchell and colleagues ${ }^{1}$ is important because existing measures of tumor immunogenicity and susceptibility to immune checkpoint inhibitors, such as the level of PD-L1 expression, fail to adequately predict response to treatment. ${ }^{2}$ Whereas the inclusion of additional indicators, such as tumor mutation burden, may improve accuracy, such analysis requires tumor genome sequencing, which is not available to all patients undergoing treatment. ${ }^{3,4}$ Therefore, Ki67 staining could be an attractive adjunct, owing to its low cost and wide availability.

Although the potential clinical implications of the identified association are promising, the mechanisms underlying

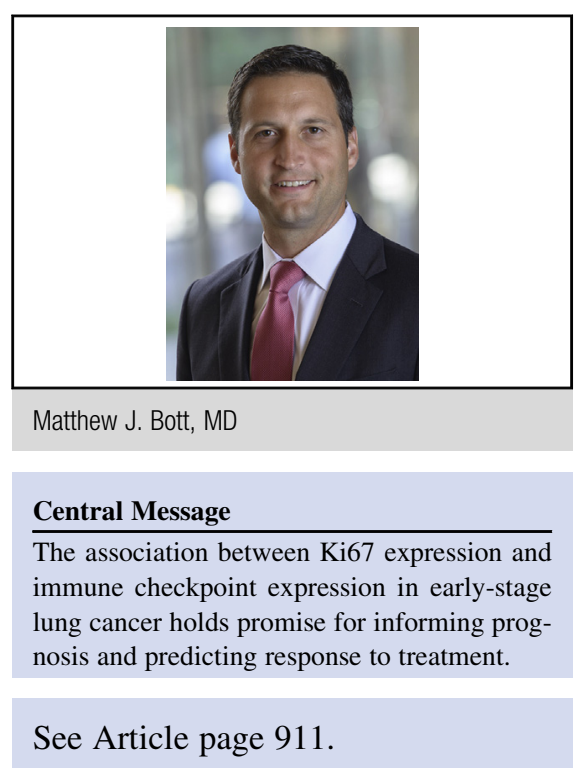

it remain unclear. Cellular pathways that are critical for proliferation, such as the mitogen-activated protein kinase (MAPK) cascade and effectors of phosphoinositide 3-kinase (PI3K), have been shown to transcriptionally regulate levels of PD-L1. ${ }^{5}$ Similarly, a recent publication suggests that, in small cell lung cancer (a tumor type with high proliferative capacity and commonly elevated Ki67 expression), DNA damage response pathways may suppress replication stress-induced expression of immunosuppressive proteins. ${ }^{6}$ Furthermore, disrupting these repair mechanisms led to increased PD-L1 expression and enhanced sensitivity to T-cell checkpoint inhibition.

Another interesting aspect of the current study is that the association between cell proliferation and an immunosuppressive microenvironment was confined to stage I tumors. In the authors' stage-specific analysis, Ki67 expression and levels of immune checkpoint expression were not significantly associated in stage II and III tumors. Although the explanation for this finding also remains obscured, it is tempting to speculate that later-stage tumors may have undergone sufficient immunoediting such that the remaining tumor cells have fewer reactive neoantigens and are therefore less reliant on the expression of immune checkpoint proteins for immune evasion.

Regardless, the association between cell proliferation and immune suppression in stage I cancers holds relevance, as stage IB tumors are often included in trials of neoadjuvant and adjuvant T-cell checkpoint inhibition in 
NSCLC. $^{7,8}$ Therefore, if Ki67 expression does predict response to therapy, this might help to select patients for this treatment approach. The authors' finding that high Ki67 expression ( $>25 \%$ of tumor cells) was associated with increased mortality may provide an additional rationale for the use of multimodality treatment in this patient population.

Mitchell and colleagues ${ }^{1}$ highlight an intriguing association between cell proliferation and an immunosuppressive microenvironment in early-stage lung cancer. If validated in additional cohorts, these findings may provide a promising avenue for predicting response to $\mathrm{T}$-cell checkpoint inhibition in patients with early-stage NSCLC. Such a strategy may ultimately allow us to expand the benefits of immunotherapy to an even larger proportion of patients with lung cancer.

\section{References}

1. Mitchell KG, Parra ER, Nelson DB, Zhang J, Wistuba II, Fujimoto J, et al. Tumor cellular proliferation is associated with enhanced immune checkpoint expression in stage I non-small cell lung cancer. J Thorac Cardiovasc Surg. 2019;158:911-9.e6.

2. Garon EB, Rizvi NA, Hui R, Leighl N, Balmanoukian AS, Eder JP, et al Pembrolizumab for the treatment of non-small-cell lung cancer. $N$ Engl J Med. 2015;372:2018-28.

3. Rizvi H, Sanchez-Vega F, La K, Chatila W, Jonsson P, Halpenny D, et al Molecular determinants of response to anti-programmed cell death (PD)-1 and anti-programmed death-ligand 1 (PD-L1) blockade in patients with non-smallcell lung cancer profiled with targeted next-generation sequencing. J Clin Oncol. 2018:36:633-41.

4. Ready N, Hellmann MD, Awad MM, Otterson GA, Gutierrez M, Gainor JF, et al. First-line nivolumab plus ipilimumab in advanced non-small-cell lung cancer (CheckMate 568): outcomes by programmed death ligand 1 and tumor mutational burden as biomarkers. J Clin Oncol. 2019;37:992-1000.

5. Wang Y, Wang H, Yao H, Li C, Fang JY, Xu J. Regulation of PD-L1: emerging routes for targeting tumor immune evasion. Front Pharmacol. 2018;9:536.

6. Sen T, Rodriguez BL, Chen L, Corte CMD, Morikawa N, Fujimoto J, et al Targeting DNA damage response promotes antitumor immunity through STING-mediated T-cell activation in small cell lung cancer. Cancer Discov. 2019;9:646-61.

7. Forde PM, Chaft JE, Smith KN, Anagnostou V, Cottrell TR, Hellmann MD, et al Neoadjuvant PD-1 blockade in resectable lung cancer. N Engl J Med. 2018;378: 1976-86.

8. Yang CJ, McSherry F, Mayne NR, Wang X, Berry MF, Tong B, et al. Surgica outcomes after neoadjuvant chemotherapy and ipilimumab for non-small cell lung cancer. Ann Thorac Surg. 2018;105:924-9. 